\title{
TRANSPLANTATION \\ Investigating the role of BAFF in kidney graft dysfunction
}

Researchers in France report that high levels of soluble B-cell activating factor (BAFF) and high levels of its receptor BAFF-R are associated with the development of donor-specific antibodies (DSAs) and with an increased risk of kidney graft dysfunction in renal transplants, respectively.

Previous studies have shown that BAFF is likely to have a role in the process of post-transplantation organ rejection, probably by acting on the process of B-cell maturation. Increased BAFF production has also been shown to be associated with the development of autoimmune disease.

In the latest study, researchers set out to determine whether a correlation exists between BAFF levels and the development of graft dysfunction or the production of donor-specific or anti-HLA antibodies in renal transplant recipients with stable renal function at baseline.

Thibault-Espitia et al. measured levels of BAFF mRNA transcripts, soluble BAFF protein, and transcripts coding for BAFF receptors (BAFF-R, TACI and BCMA) in the blood of 143 renal transplant recipients who were on standard immunosuppressive therapy and had demonstrated stable graft function for at least 5 years.

During the follow-up period of 6-8 years after study inclusion, renal graft dysfunction occurred in 21 patients. The researchers found that the risk of renal dysfunction was 3.6-fold higher in patients with a BAFF-R expression level of $>0.10$ than in those with a lower level. In addition, patients with decreased levels of $B A F F$ transcripts and patients with increased levels of soluble BAFF were shown to have a fourfold and a nearly fivefold increased risk of developing DSAs, respectively.

"Our results are novel as they enable us, for the first time, to predict deterioration of renal function-that was stable at the time of the initial measurements-over time," says corresponding author Sophie Brouard. "The association of a high

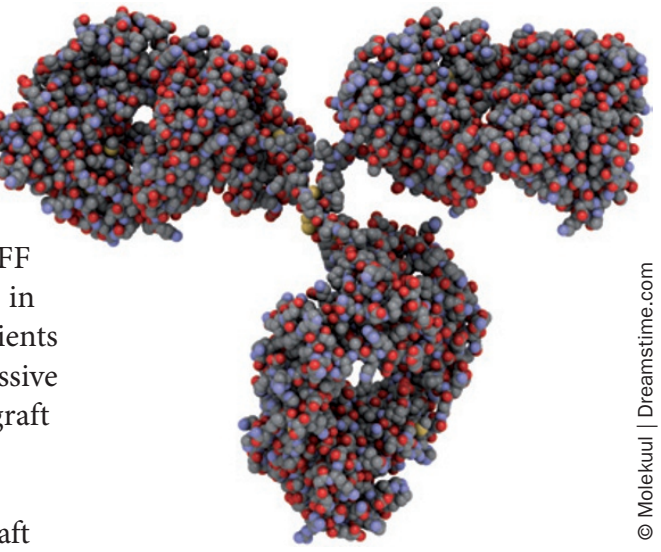

soluble BAFF level with a higher risk of de novo appearance of DSAs and a high BAFF-R level with a higher risk of renal dysfunction suggests that BAFF pathway modulation may be a risk factor for renal dysfunction, a finding that may enable pathology to be detected earlier, before the onset of clinical symptoms."

Rebecca Kelsey

Original article Thibault-Espitia, A. et al. BAFF and BAFF-R levels are associated with risk of long-term kidney graft dysfunction and development of donor-specific antibodies. Am. J. Transplant. doi:10.1111/j.1600-6143.2012.04194 